\title{
STRUCTURAL INVESTIGATIONS INTO THE CATALYTIC MECHANISM OF HUMAN MANGANESE SUPEROXIDE DISMUTASE USING NEUTRON AND X-RAY CRYSTALLOGRAPHY
}

\author{
Jahaun Azadmanesh ${ }^{1}$, William E. Lutz ${ }^{1}$, Scott R. Trickel ${ }^{1}$, Kevin L. Weiss ${ }^{2}$, Leighton Coates ${ }^{2}$, and \\ Gloria E. O. Borgstahl ${ }^{1^{*}}$ \\ 1 The Eppley Institute for Research in Cancer and Allied Diseases, 987696 Nebraska Medical Center, Omaha, NE 68198- \\ 6805, USA \\ ${ }^{2}$ Biology and Soft Matter Division, Oak Ridge National Laboratory, 1 Bethel Valley Road, Oak Ridge, TN 37831, USA
}

\section{Background and Significance}

Superoxide dismutases (SODs) are necessary antioxidant enzymes that protect cells from excessive amounts of reactive oxygen species (ROS), with deleterious effects to their catalysis causing significant phenotypic consequences. SODs perform their bio-protective role by converting superoxide, a ROS, into oxygen and hydrogen peroxide by cyclic oxidation and reduction reactions with the active site metal, which is dependent on two proton transfers. Mutations of SODs have been linked to cancer of the lung, colon, and lymphatic system, and to neurodegenerative diseases, such as Parkinson's disease and amyotrophic lateral sclerosis. While SODs have proven to be of significant biological importance since their discovery in 1968, the mechanistic nature of their catalytic function remains elusive. Extensive investigations with a multitude of approaches have been employed to unveil the catalytic workings of SODs, but experimental limitations have impeded direct observations of the mechanism. The proton-based catalytic mechanism remains unclear.

\section{Experiments and Results}

How superoxide interacts with the catalytic site has been difficult to investigate owing to the fast catalytic rate of SODs and the short half-life of superoxide. In our crystallographic study, we instead used azide, a substrate analog, to visualize a model of superoxide binding to MnSOD. Additionally, electrostatic surfaces of MnSOD in several possible catalytic states were generated to study the manner of superoxide diffusion to the active site. Given that superoxide is negatively charged, charged surfaces of MnSOD detail the path of substrate diffusion to the active site.

For MnSOD, our group has recently proposed a proton relay structural model, consisting of specific amino acid residues and water molecules, to be the source of protons for proton-coupled electron transfer at the manganese active site. We are using neutron diffraction to test this catalytic model by visualizing the protonation states along the relay, which has not been possible with the countless X-ray structures published during the last 20 years. Our methods for growth of large $\left(>0.2 \mathrm{~mm}^{3}\right)$, perdeuterated crystals of MnSOD needed for neutron diffraction, to a resolution where hydrogen positions can be visualized $(<2.5 \AA)$, has been refined to the extent that the crystals can be produced consistently and reliably. These crystals are inherently challenging for neutron diffraction because they have large unit cell dimensions $(a=b=80 \AA, c=240 \AA)$, which significantly decreases the signal. However, our work using the Macromolecular Neutron Diffractometer at Oak Ridge National Laboratory provides the largest unit cell ever to have yielded neutron data to sufficient resolution where hydrogen positions can be visualized, expanding the possibilities of neutron crystallography.

We sought to obtain neutron data on fully oxidized and fully reduced crystals as protonations at the catalytic site of MnSOD are hypothesized to differ based on the redox status of the manganese. An approach was devised in which the redox state of perdeuterated crystals could be controlled and changed within seconds, with minimal compromise of crystal integrity. Data collection and refinements of the oxidized and reduced structures reveal 3D visualization of protonation positions. At the catalytic site, several molecules are protonated and some protonation states differ based on the redox status. These structures unveil a proton relay for proton-assisted electron transfer where a [model] catalytic mechanism can be deduced. 